\title{
Synthetic Aperture Sequential Beamforming using Spatial Matched Filtering
}

Schou, Mikkel; di lanni, Tommaso; Bouzari, Hamed; Jensen, Jørgen Arendt

Published in:

Proceedings of 2017 IEEE International Ultrasonics Symposium (IUS)

Publication date:

2017

Document Version

Peer reviewed version

Link back to DTU Orbit

Citation (APA):

Schou, M., di lanni, T., Bouzari, H., \& Jensen, J. A. (2017). Synthetic Aperture Sequential Beamforming using Spatial Matched Filtering. In Proceedings of 2017 IEEE International Ultrasonics Symposium (IUS) IEEE.

\section{General rights}

Copyright and moral rights for the publications made accessible in the public portal are retained by the authors and/or other copyright owners and it is a condition of accessing publications that users recognise and abide by the legal requirements associated with these rights.

- Users may download and print one copy of any publication from the public portal for the purpose of private study or research.

- You may not further distribute the material or use it for any profit-making activity or commercial gain

- You may freely distribute the URL identifying the publication in the public portal

If you believe that this document breaches copyright please contact us providing details, and we will remove access to the work immediately and investigate your claim. 


\title{
Synthetic Aperture Sequential Beamforming using Spatial Matched Filtering
}

\author{
Mikkel Schou, Tommaso di Ianni, Hamed Bouzari, and Jørgen Arendt Jensen, \\ Center for Fast Ultrasound Imaging, Department of Electrical Engineering, \\ Technical University of Denmark, DK-2800 Lyngby, Denmark
}

\begin{abstract}
Synthetic Aperture Sequential Beamforming (SASB) has shown to achieve a good resolution and high penetration depth. The low complexity at the transducer level of the beamformer makes it ideal for use with a handheld device. SASB with a low $F \#(\leq \mathbf{0 . 5})$ can achieve even better resolution at the cost of high grating lobes, which causes loss of contrast in the final image. In this paper, Spatial Matched Filtering (SMF) was used instead the second stage of beamformer, in an attempt to suppress the grating lobes. The advantage of SMF over SASB was investigated by pushing the limits of $F \#$, from 1.5 to 0.5 . The effect of the number of emissions used in first stage was also investigated. A $3.3 \mathrm{MHz}$ BK Ultrasound 9040 convex array was simulated in Field II on a point scatter phantom and a cyst phantom. The resolution was quantified with the full-widthhalf-max (FWHM), and the contrast was measured with the $20 \mathrm{~dB}$ cystic resolution. The contrast-to-noise ratio (CNR) was calculated for the cyst mimicking phantom. The results showed that SMF achieved similar resolution as SASB and improved grating lobe suppression leading to an increase in contrast. The grating lobes caused by an $F \#$ of 0.5 are dominant in the SASB images, but not as much in SMF images. The CNR for a cyst mimicking phantom was improved $7 \mathrm{~dB}$ and $6 \mathrm{~dB}$ for SMF over SASB at depth $20 \mathrm{~mm}$ and $30 \mathrm{~mm}$, with an $F \#$ of 0.5 and 256 emissions. The FWHM for SMF was slightly higher than SASB across all depth and parameter settings, with a maximum difference of $0.3 \mathrm{~mm}$. It was demonstrated that SMF can achieve similar resolution to SASB and for certain parameter settings improve the contrast by suppressing the grating lobe artifacts.
\end{abstract}

\section{INTRODUCTION}

Synthetic Aperture Sequential Beamforming (SASB) has shown great potential for use in a handheld device setup [1], [2]. In some cases it even outperforms the conventional Dynamic Receive focusing (DRF) [1]. SASB is a two stage beamforming approach. The first stage is handled at the transducer level with a fixed received beamformer, where the time delay profile and apodization weights are fixed. The focusing scheme is thereby simplified compared to DRF, where the receive delay profile changes dynamically as a function of depth. The output from the first stage beamformer is a single time signal that can be passed from the first to the second stage. The data required to sample changes from $N$ receiver channel signals to a single signal. The second stage beamformer utilizes the Time of Flight (TOF) of the emitted wave to beamform a single Low Resolution Image $(L R I)$. The obtained resolution is proportional to the wavelength times the $F \#$ and lowering the $F \#$ directly increases the resolution. This, however, affects the contrast negatively, as it results in higher energy of the side lobes and in off center grating lobes [2]. Usually an $F \# \geq 2$ is used to avoid these [1]. The performance of the system depends on many parameters, but the two most influencial have proven to be the $F \#$ combined with the spatial sampling, which is determined by the number of first stage lines, i.e. number of emissions [2].

The method assumes the spatial impulse response to be a delta function for all the spatial positions. However, this assumption is not valid, since it does not account for the shape of the emitted beam, spatial extent of the transducer elements, or that the received response will vary as a function of spatial position [3]. Spatial Matched Filtering (SMF) can be applied to account for the spatial variations of the impulse response. SMF beamforming have proved to lower unwanted artifacts and increase the SNR, by aligning the responses as a function of spatial position [4].

In this paper, an SMF approach is used in place of the second stage beamformer. The filters are calculated for every point in the field-of-view (FOV) and applied to the first stage beamformed lines, i.e. the Low Resolution Lines (LRL). This preserves the low complexity of the first stage beamformer. The concept of SASB and SMF will be introduced in the following two sections. Later the proposed SMF algorithm will be investigated with the use of simulations.

\section{A. Synthetic Aperture Sequential Beamforming (SASB)}

SASB synthetizes a virtual aperture by applying a Fixed Receive Focusing (FRF) scheme as a first stage beamformer. The output from the first stage beamformer is a low resolution line $(L R L)$. The transmission origin $\left(\vec{r}_{\theta_{n}}\right)$ and the fixed focal point, i.e. virtual sources $\left(\vec{r}_{v S_{n}}\right)$ are then used to generate an image based on the wave path of the emitted wave [2]. The sequential aspect of SASB is achieved by generating a single 2D low resolution image (LRI) for emission $n$. Adding the next LRI for $n+1$ reveals a HRI that is updated sequentially, as the next $L R L$ is passed through the second stage beamformer. The FRF scheme result in a limited area being hit by the emitted wave. This can be seen in Fig. 1a An image point at $\vec{r}_{i}$ is therefore represented in emission 1 and 2, but will not be present in 3. By changing $\vec{r}_{\theta_{n}}$ and $\vec{r}_{v s_{n}}$ between emissions generates the synthetic aperture at positions $\vec{r}_{v s_{n}}$ for $n=1: N$, where $N$ is the total number of emissions used in the first stage to cover a full scan sector. This has previously been described for a convex transducer array [1]. 


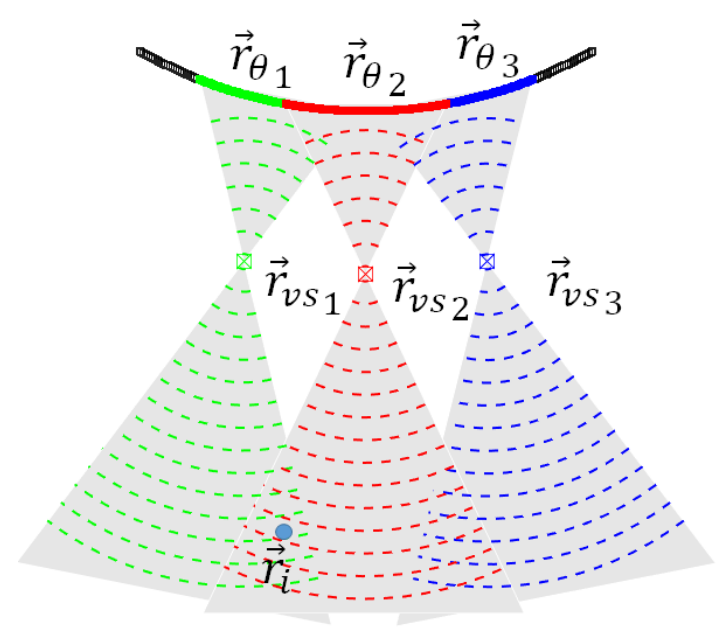

(a) Geometry of three emissions

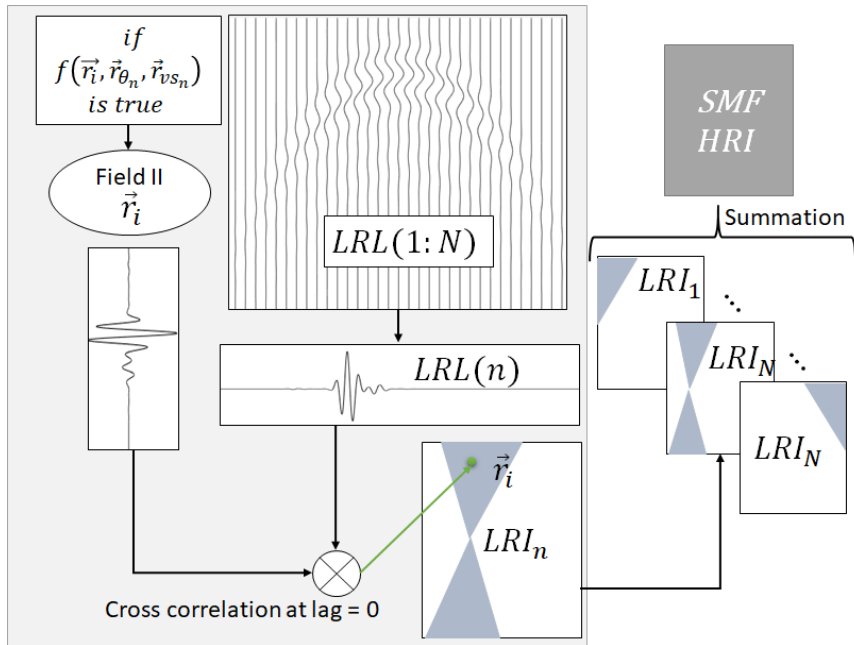

(b) Flowdiagram of algorithm

Fig. 1. a) shows the geometry of three emissions, with virtual source $\vec{r}_{v s_{n}}$, transmit origin $\vec{r}_{\theta_{n}}$ and imagepoint $\vec{r}_{i}$. b) Shows the flowdiagram of the SMFalgorithm. $f$ is a boolean expression which determine whether or not, the image point $r_{i}$ is inside the insonified area of emission $n$. The process is repeated until all discrete image points inside the blue area are processed. The $L R I(n)$ are then added to the last $L R I(1: n-1)$ to form a HRI.

\section{B. Spatial Matched Filtering (SMF)}

The second stage of SASB can be substituted with an SMF approach. The first stage consist of a FRF beamformer like the conventional SASB [1], [2]. Similarly, only the insonified area seen in Fig. 1a is considered as targets for filtration. For each point inside the insonified area, the response of each FRF beamformer are matched filtered using a time-reversed version of the expected response. Finally the SMF responses of $N$ emissions are summed to reveal the final HRI. The final SMF HRI can be sequentially updated, by repeating the emissions scheme. A graphical representation of the algorithm is found in Fig. $1 b$ The method assumes the linear ultrasound model is valid and that the optimal filter is given by the time-reversed response from a spatial impulse, showed by [3] as:

$$
p_{r}\left(\vec{r}_{1}, \vec{r}_{2}, t\right)=v_{p e}(t) \underset{t}{\star} h_{t}\left(\vec{r}_{1}, \vec{r}_{2}, t\right) \underset{t}{\star} h_{r}\left(\vec{r}_{2}, \vec{r}_{1}, t\right) .
$$

$p_{r}$ is the expected response used to filter the signals. It is defined as the electro-mechanical impulse response of the transducer $v_{p e}(t)$, and the impulse response during transmission $h_{t}(t)$ and reception $h_{r}(t)$. The impulse responses are dependent on the transmitter location $\vec{r}_{1}$ and receiver element location $\vec{r}_{2}$. To emphasize that the filters are applied after the fixed receive beamformer, a change of notation is performed. The filter operation therefore becomes:

$$
\operatorname{HRI}\left(\vec{r}_{i}\right)=\sum_{n=1}^{N} \int_{t_{i}}^{t_{i}+\Delta T_{i}} L R L_{n}(t) \cdot E_{F R F, n}\left(\vec{r}_{i}, t\right) d t .
$$

Here $H R I$ is the high resolution image with spatial position $\vec{r}_{i}$ defined by the image grid coordinates with a specified FOV and spatial sampling density. $t_{i}$ is the start time of $E_{F R F}\left(\vec{r}_{i}, t\right)$ and $\Delta T_{i}$ is the response length. The points in (2) are calculated in a grid corresponding to the pixels of the final image, and therefore no scan conversion is required. $E_{F R F, n}\left(\vec{r}_{i}, t\right)$ is the expected response from a "true" scatter point placed at $\vec{r}_{i}$ and also accounts for the effect of the transducer array geometry including their focusing and apodization.

The output from the first stage beamformer, i.e. $L R L$ acquired from emission $n$, is denoted noted $L R L_{n} . \vec{r}_{\theta_{n}}$ and $\vec{r}_{v S_{n}}$ can be changed with a sliding aperture approach. The time delay and apodization weighs used to beamform $L R L_{n}$ should therefore match the exact same as $E_{F R F, n} . t_{i}$ and $\Delta T_{i}$ are provided, when $E_{F R F, n}$ are estimated. $E_{F R F, n}$ can be estimated with a simulation or experimentally recorded. For each emission $n$ a total of $M$ simulations are required, where $M$ is the number of image points in the defined image grid.

\section{METHOD}

The model used in this paper was the BK9040 convex transducer array. The method is easily translatable to other transducer geometries. The developed algorithm was tested with simulations performed with Field II [5], [6]. The phantoms used consisted of a point scatter phantom and a cystmimicking phantom. To preserve the portable aspect of SASB, the second stage of SASB was substituted with an SMF approach. SMF was applied on the LRLs, to ensure the low complexity of the first stage beamformer. This ensures only one signal is passed from the first to the second stage per emission, similar to SASB. The enhanced resolution with a lower $F \#$ for SASB was the foundation of exploring the feasibility of applying SMF. To utilize the gain in resolution, the side and/or grating lobes should be decreased or completely removed.

\section{A. Simulation Setup and Image Performance estimators}

$E_{F R F, n}$ for the SMF algorithm were simulated with Field II, providing the match filter coefficients. The simulations used to test the algorithm were likewise simulated with Field II. The 


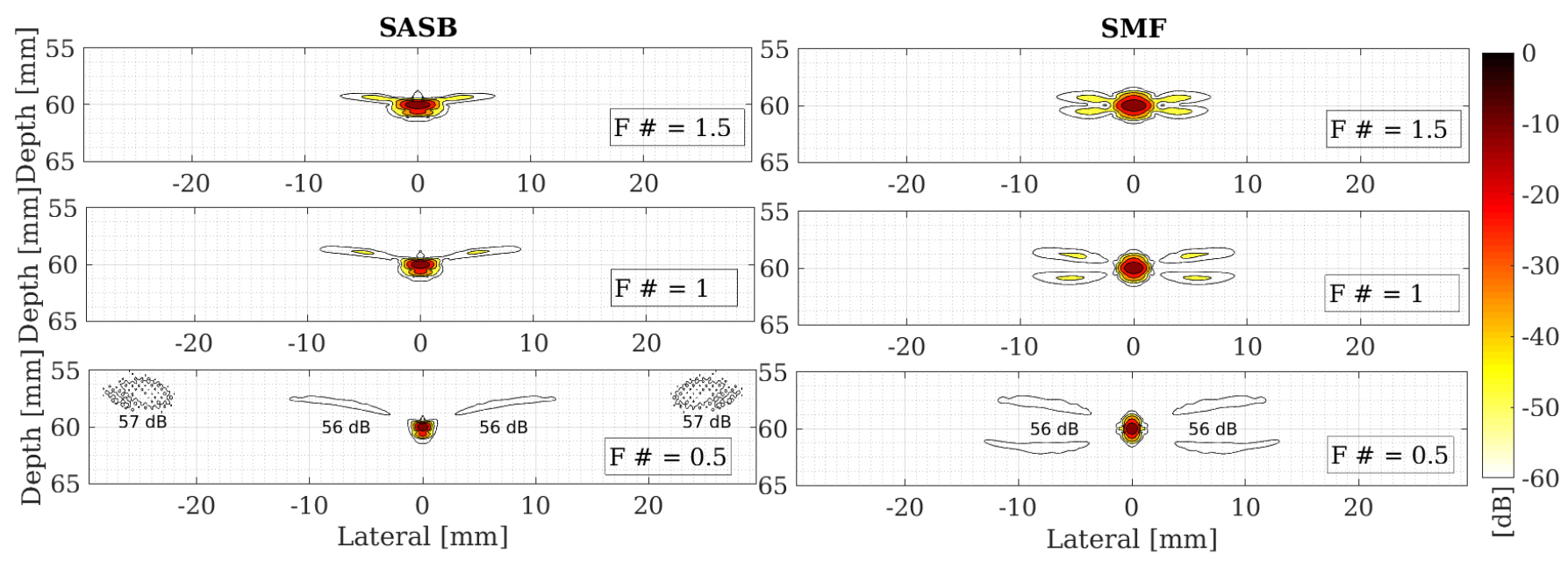

Fig. 2. Left panel: shows contour plot of PSF at $60 \mathrm{~mm}$ imaged with SASB, for different $F \#$. Right panel: contour of PSF at $60 \mathrm{~mm}$ imaged with SMF, for different $F \#$. All were acquired with 384 emissions. The contour lines are shown with a spacing of $12 \mathrm{~dB}$, and the side lobe and grating lobe peak values are reported for $F \#=0.5$.

model parameters and transducer setting used for SASB, SMF, and DRF are shown in Table I The two parameters: $N$ and $F \#$ were changed to test the limit of the two beamformers. In total two phantoms were simulated, a point scatter phantom with point scatters placed as a function of depth, and a cyst-micking phantom used to estimate the contrast-to-noise ratio (CNR) [7]. A conventional DRF image of the point scatter phantom was also generated as a reference for the two others beamformers. The CNR was therefore calculated as: $C N R=20 \log \left(\left|\mu_{\text {back }}-\mu_{\text {cyst }}\right| / \sqrt{\sigma_{\text {back }}^{2}+\sigma_{c y s t}^{2}}\right)$, with $\mu_{\text {back }}$ and $\mu_{\text {cyst }}$ as the mean signal intensity of background and cyst respectively. $\sigma_{b a c k}^{2}$ and $\sigma_{c y s t}^{2}$ are the variance of the background and cyst intensity. Main to grating lobe level difference (M2Gp), a measure of the grating lobe suppression, was introduced as:

$$
\mathrm{M} 2 \mathrm{Gp}=P_{\text {main lobe }}(R)-E\left\{P_{\text {grating lobe }}(R)\right\} .
$$

With peak level energy $P_{\text {main lobe }}$ of the main lobe extracted from the profile with radius $R$ from the transducer surface. $E\left\{P_{\text {grating lobe }}(R)\right\}$ symbolizes the mean of the two grating lobes peak levels. It is visualized in Fig. 3 at the bottom.

\section{RESULTS}

The current form of the SMF algorithm revealed higher sidelobes, as seen by the mirrored side lobes in Fig. 2. Here contour plot of the Point Spread function at $60 \mathrm{~mm}$ is seen. Grating lobes were present for an $F \#$ of 0.5 for SASB and suppressed with SMF. The M2Gp measure revealed a higher grating lobe suppression for $N=384$ and $F \#$ of 0.5 , as seen in Fig 3 a. The quantified lateral $\mathrm{FWHM}_{x}$ and $20 \mathrm{~dB}$ cystic resolution $(\mathrm{CR} 20 \mathrm{db})$ are shown in Fig. 3 b. These revealed that both SASB and SMF follows the same pattern, of increased resolution with decreasing $F \#$. The largest difference between the two were $0.3 \mathrm{~mm}$ and occurred at $30 \mathrm{~mm}$ for $F \#$ of 1.5, i.e. the focal point of the FRF in the first stage. Both SASB and
TABLE I

TRANSDUCER PARAMETERS, SCAN SETTINGS \& CONSTANTS

\begin{tabular}{|c|c|c|c|}
\hline Parameter & \multicolumn{3}{|c|}{ BK } \\
\hline Number of elements & \multicolumn{3}{|c|}{192} \\
\hline Center frequency & \multicolumn{3}{|c|}{$3.30 \mathrm{MHz}$} \\
\hline Bandwidth & \multicolumn{3}{|c|}{$90 \%$} \\
\hline Pitch & \multicolumn{3}{|c|}{$0.330 \mathrm{~mm}$} \\
\hline Height of element & \multicolumn{3}{|c|}{$13.0 \mathrm{~mm}$} \\
\hline Elevation focus & \multicolumn{3}{|c|}{$70.0 \mathrm{~mm}$} \\
\hline Radius of curvature & \multicolumn{3}{|c|}{$60.25 \mathrm{~mm}$} \\
\hline Kerf & \multicolumn{3}{|c|}{$0.01 \mathrm{~mm}$} \\
\hline Speed of Sound & \multicolumn{3}{|c|}{$1540 \mathrm{~m} / \mathrm{s}$} \\
\hline \multicolumn{4}{|c|}{ SASB/SMF 1st stage } \\
\hline Apodization type (Tx/Rx) & \multicolumn{3}{|c|}{ Hamming } \\
\hline No. of emissions (varying) & \multicolumn{3}{|c|}{$192-384$} \\
\hline Scan sector & \multicolumn{3}{|c|}{$60.5^{\circ}$} \\
\hline No. of active elements & \multicolumn{3}{|c|}{64} \\
\hline$F \#(\mathrm{Tx} / \mathrm{Rx})$ & $1.5 / 1.5$ & $1 / 1$ & $0.5 / 0.5$ \\
\hline Focal Length $(\mathrm{Tx} / \mathrm{Rx})$ & $31 \mathrm{~mm}$ & $21 \mathrm{~mm}$ & $11 \mathrm{~mm}$ \\
\hline \multicolumn{4}{|c|}{ DRF } \\
\hline$F \#(\mathrm{Tx} / \mathrm{Rx})$ & \multicolumn{3}{|c|}{$4.5 / 0.8$} \\
\hline focal Length (mm) & \multirow{2}{*}{\multicolumn{3}{|c|}{$\begin{array}{l}40 \mathrm{~mm} / \text { dynamic } \\
\text { Rect / Hamming }\end{array}$}} \\
\hline Apodization type (Tx/Rx) & & & \\
\hline No. emissions & \multicolumn{3}{|c|}{192} \\
\hline
\end{tabular}

SMF outperforms DRF in FWHM $x$ for $F \# \leq 1$. The CR20dB for $F \#=0.5$ is comparable to DRF. Due to the hamming apodization, the side lobes are quite dominant when the $F \#$ is lower than 1 resulting in a higher CR20dB. The mirrored side lobes of SMF provided the generally higher CR20dB for SMF compared to SASB. The cyst phantom was imaged with SASB and SMF in Fig. 4. The CNR was quantified for the cyst at 20 and $30 \mathrm{~mm}$ depth. For SMF, a CNR of $8.21 \mathrm{~dB}$, and $2.70 \mathrm{~dB}$, compared with $1.12 \mathrm{~dB}$, and $-3.90 \mathrm{~dB}$ provided by SASB. This gives a CNR improvement of approximately $7 \mathrm{~dB}$ at $20 \mathrm{~mm}$, and $6 \mathrm{~dB}$ at $30 \mathrm{~mm}$. The processing time on a Intel Core i7-3770 CPU @ 3.40GHz x 8 CPU, with $7.8 \mathrm{~Gb}$ ram provided an increase in processing time of 45 minutes of SMF in Matlab, compared to SASB for the images in Fig 4 


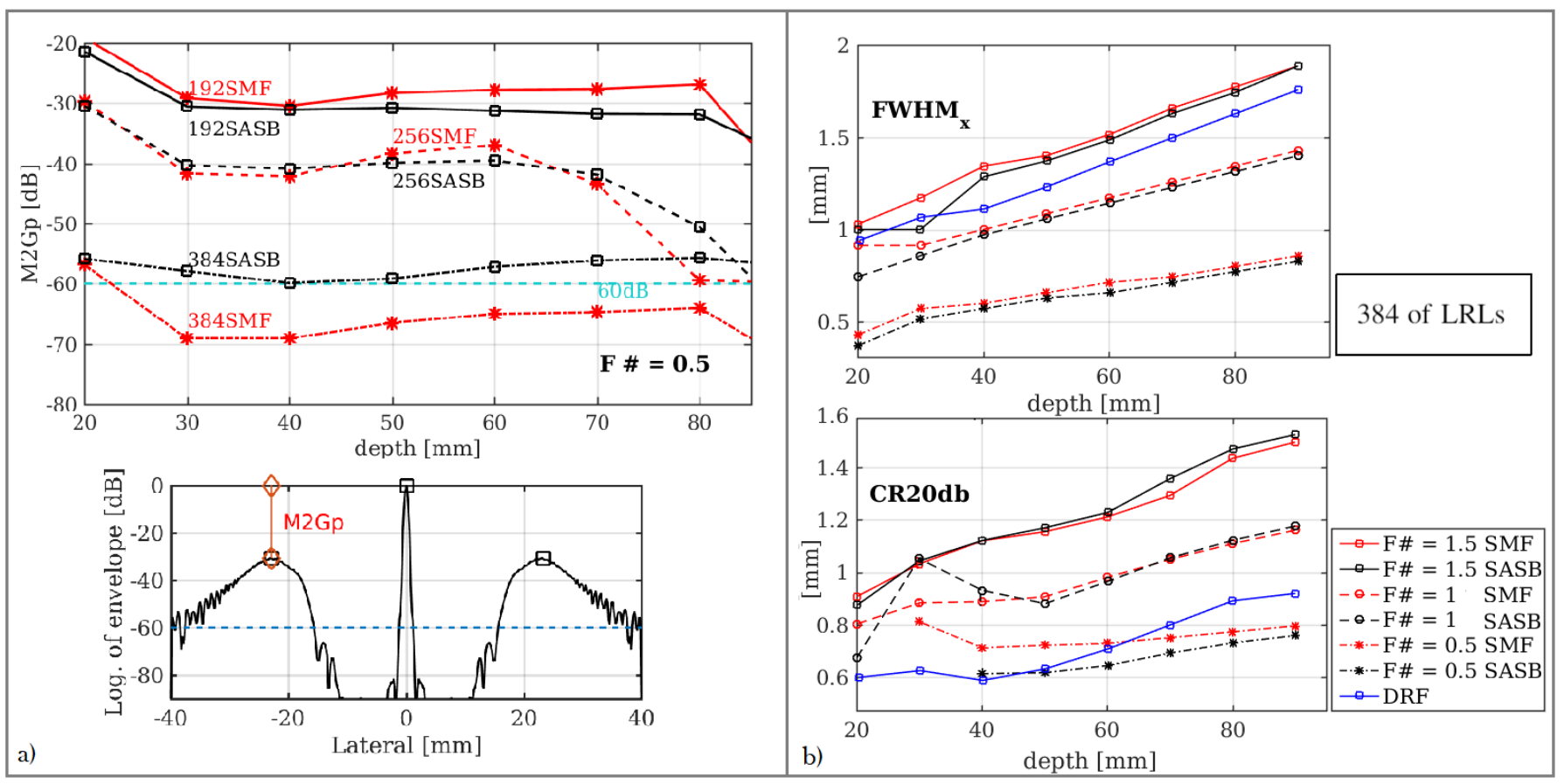

Fig. 3. a): top shows the measured M2Gp for $\mathrm{N}=192,256,384$ with $F \#$ of 0.5 , and bottom shows how the measure is extracted from a PSF profile at radius $R$ from the transducer surface. b): Shows the calculated $\mathrm{FWHM}_{x}$ and CR20dB for SMF and SASB with $F \#=1.5,1,0.5$. SMF is shown with red, SASB with black, and DRF is shown with blue color.

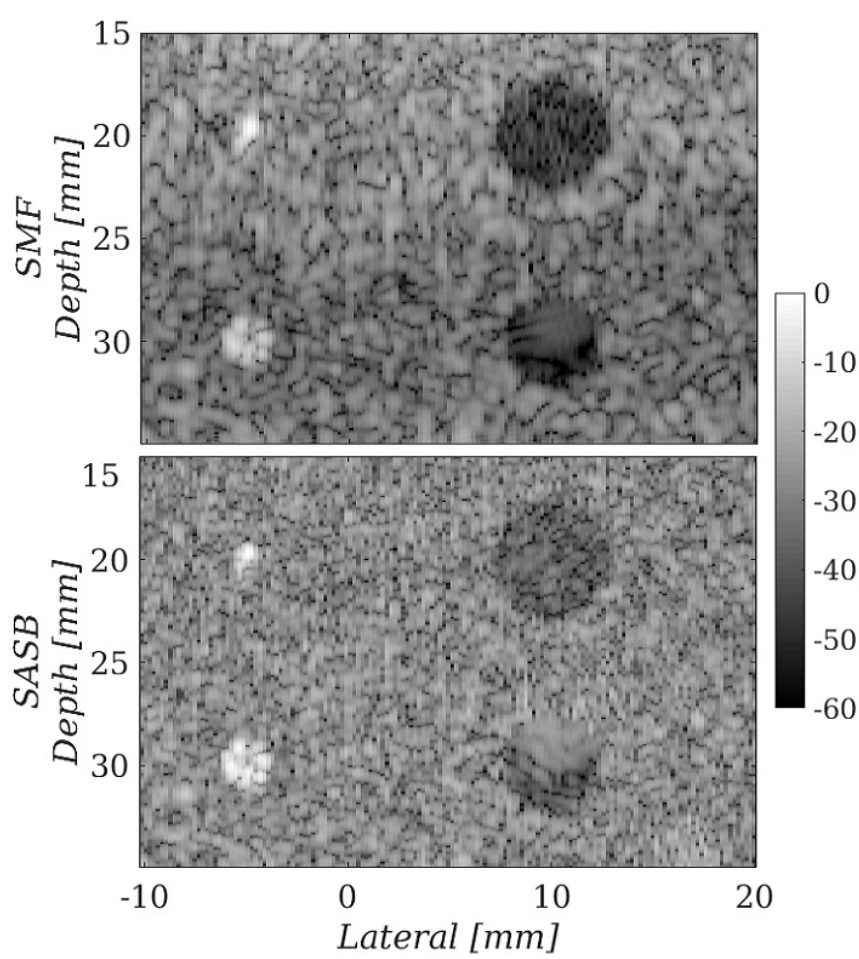

Fig. 4. Both images were made of the same Field II simulated data originating from a cyst mimicking phantom. Top image shows SMF, bottom image shows SASB. Dynamic range is $60 \mathrm{~dB}$. Images were acquired with 256 emissions, $F \#$ of 0.5 and a scan sector of $60^{\circ}$.

\section{CONCLUSION}

In this paper we demonstrate that an SMF approach can be successfully used in place of the second stage beamformer of a synthetic aperture approach with simulations. The resolution proved to be similar to standard SASB for the given parameters. An improved contrast can be achieved with SMF over SASB for an $F \#$ of 0.5 in simulations. The area of application depends on the purpose, and the higher processing time has to be considered, when deciding between SASB and SMF. Simulation and the correlation computation time should be considered as obstacles for a commercial implementation of the method.

\section{REFERENCES}

[1] M. C. Hemmsen, P. M. Hansen, T. Lange, J. M. Hansen, K. L. Hansen, M. B. Nielsen, and J. A. Jensen, "In vivo evaluation of synthetic aperture sequential beamforming," Ultrasound Med. Biol., vol. 38, no. 4, pp. 708716, 2012.

[2] J. Kortbek, J. A. Jensen, and K. L. Gammelmark, "Sequential beamforming for synthetic aperture imaging," Ultrasonics, vol. 53, no. 1, pp. 1-16, 2013.

[3] J. A. Jensen and P. Gori, "Spatial filters for focusing ultrasound images," in Proc. IEEE Ultrason. Symp., 2001, pp. 1507-1511.

[4] K. S. Kim, J. Liu, and M. F. Insana, "Efficient array beam forming by spatial filtering for ultrasound B-mode imaging," J. Acoust. Soc. Am., vol. 120 , no. 2, pp. 852-861, 2006.

[5] J. A. Jensen and N. B. Svendsen, "Calculation of pressure fields from arbitrarily shaped, apodized, and excited ultrasound transducers," IEEE Trans. Ultrason., Ferroelec., Freq. Contr., vol. 39, pp. 262-267, 1992.

[6] J. A. Jensen, "Field: A program for simulating ultrasound systems," Med. Biol. Eng. Comp., vol. 10th Nordic-Baltic Conference on Biomedical Imaging, Vol. 4, Supplement 1, Part 1, pp. 351-353, 1996.

[7] H. Bouzari, M. Engholm, T. L. Christiansen, C. Beers, A. Lei, M. B. Stuart, S. I. Nikolov, E. V. Thomsen, and J. A. Jensen, "Volumetric synthetic aperture imaging with a piezoelectric 2-D row-column probe," in Proc. SPIE Med. Imag., vol. 9790, 2016, pp. 1-9. 